\author{
Chung-Jen Lu ${ }^{1}$ \\ Associate Professor \\ e-mail: cjlu@ntu.edu.tw \\ Chia-Hsing Hung \\ Graduate Student \\ Department of Mechanical Engineering,
National Taiwan University,
No. 1 Roosevelt Road Section 4,
Taipei 10617, Taiwan, R.0.C.
}

\title{
Stability Analysis of a Three-Ball Automatic Balancer
}

Ball-type automatic balancers can effectively reduce the vibrations of optical disk drives due to the inherent imbalance of the disk. Although the ball-type automatic balancer used in practice consists of several balls moving along a circular orbit, few studies have investigated the dynamic characteristics of ball-type balancers with more than two balls. The aim of this paper is to study the dynamic characteristics of a three-ball automatic balancer. Emphasis is put on the effects of the number of balls on the stability of the perfect balancing positions - the equilibrium positions where the disk is perfectly balanced. A theoretical model of an optical disk drive packed with a three-ball automatic balancer is constructed first. The governing equations of the theoretical model are derived using Lagrange's equations. Closed-form formulas for the equilibrium positions are presented. The stability of the perfect balancing positions is checked with the variations for a pair of design parameters. Stable regions of the perfect balancing positions in the parameter plane of a three-ball balancer are identified and compared with those of a two-ball balancer. [DOI: 10.1115/1.2948415]

\section{Introduction}

Optical disk drives have been widely used for data storage. The speed of the spindle has been brought up to $10,000 \mathrm{rpm}$ to increase the data transfer rate. At such a high rotational speed, the optical disk drive may suffer from serious vibrations due to the eccentricity of the optical disk used. Since the imbalance varies from disk to disk, it is desirable to have an automatic balancer system (ABS) equipped with an optical disk drive that can eliminate the imbalance associated with each disk automatically. The most popular ABS adopted by optical disk drive industry is the ball-type ABS. A ball-type ABS consists of several balls moving along a circular orbit. Under proper working conditions, the balls will settle at specific positions such that the vibration due to the eccentric mass of the disk can be totally suppressed. These specific positions are referred to as the perfect balancing positions henceforth. The performance of the ABS is closely related to the stability of the perfect balancing positions.

Bövik and Högfors [1] analyzed the stability of the perfect balancing position of a two-ball balancer using the method of multiple scales. Lee and Van Moorhem [2] studied the stability of the equilibrium positions of a one-ball ABS using the Floquet theory. Rajalingham et al. [3] investigated the nonlinear system consisting of an undamped rotor and a one-ball ABS. The stability of the equilibrium position was determined by the corresponding linearized system. Hwang and Chung [4] studied the dynamic characteristics of a two-ball balancer with double races. Chung and Ro [5] and Kang et al. [6] investigated the stability of a two-ball ABS comprehensively. The stability was checked with the variations for a pair of design parameters. Kim and Chung [7] employed the Floquet theory to study the effects of the stiffness and damping of rubber suspensions on the stability of the equilibrium positions. Huang et al. [8] investigated the performance of a one-ball ABS on the radial vibration reduction of optical drives. $\mathrm{Lu}$ [9] analytically studied the stability of the equilibrium positions of a one-ball balancer. The results indicated that at most one stable equilibrium state may exist at a rotating speed. Wang and $\mathrm{Lu}$ [10] proposed a new design of ball-type balancer that may increase the stable region of the perfect balancing position. Chung and Jang [11] con-

\footnotetext{
${ }^{1}$ Corresponding author.

Contributed by the Technical Committee on Vibration and Sound of ASME for publication in the Journal of Vibration AND Acoustics. Manuscript received June 4, 2007; final manuscript received April 1, 2008; published online August 14, 2008. Assoc. Editor: Weidong Zhu.
}

sidered the effects of the flexibility of the shaft on the performance of the ABS. The stability of the balanced equilibrium state of a two-ball ABS was analyzed by perturbation methods. Kim et al. [12] examined the dynamic behavior of a two-ball balancer based on a three-dimensional model. Chao et al. $[13,14]$ presented a nonplanar model for a spindle-disk system with an ABS. Their results indicated that the ABS can effectively reduce the tilting angle as well as the level of radial vibration. Chao et al. [15] evaluated the performance of a two-ball ABS with consideration to the in-plane rotational motions. The effects of the nonlinearity of the suspension washers on the performance of the automatic balancer were examined in Ref. [16]. Rajalingham and Bhat [17] and Green et al. [18] found that the system may settle to a periodic attractor in addition to a balanced state. A nonlinear bifurcation analysis of a two-ball balancer for rotating machines was conducted by Green et al. [19]

Although the ball-type balancer currently used in practice consists of more than two balls, much work has been done on the dynamic characteristics of one-ball or two-ball balancers. In the present paper, we study the stability of the perfect balancing positions of a rotating disk equipped with a three-ball ABS. The nonlinear equations of motion are derived using Lagrange's equations with respect to a corotating coordinate system. Closed-form formulas for the equilibrium positions are derived. Stable regions of the perfect balancing positions in a parametric plane are identified and compared with those of a two-ball balancer.

\section{Mathematical Model and Governing Equations}

The frictional force due to the relative motion between the balls and the orbit plays an important role in determining the performance of the ABS. Three different models of frictional force on the balls have been employed in the literature: (1) viscous damping force $[1,2,4,5,7,9,11-14,17-19]$, (2) dry friction [20,21], and (3) rolling friction $[6,8,13,15,16,22]$. The major difference between these models is that (1) has no effects on the equilibrium positions while (2) and (3) can prevent the balls from settling at the desired balancing positions. When the frictional force is small, analytical studies show that these three models result in similar qualitative behaviors in the steady state. Moreover, experimental results conducted with optical disk drives agree well with the analytical predictions based on the viscous damping model $[2,8,14]$. These results imply that the viscous damping model not only greatly simplifies the analysis work but also provides useful insights for the dynamical characteristics of ball-type balancers 


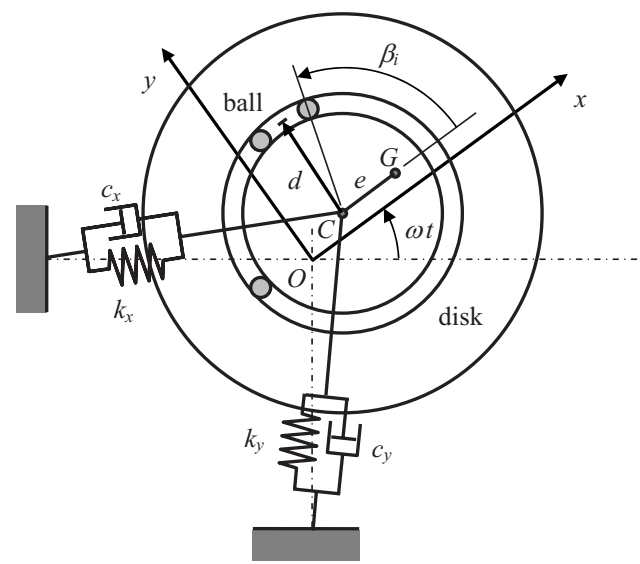

Fig. 1 Schematic of the ABS-rotor system and corotating reference frame

used in practice. Therefore the viscous damping model is employed in this work to account for the energy dissipation due to the frictional force between the balls and the orbit.

Figure 1 shows the schematic of the ABS-rotor system and the corotating reference frame. The ABS is composed of a circular disk with a groove containing balls and a damping fluid whose damping constant is $C_{B}$. The balls, each of mass $m_{b}$, move freely along the groove, subjected to viscous damping only. The radius of the groove is $d$. The product of $m_{b}$ and $d$ is the amount of imbalance caused by a single ball. For simplicity, $m_{b} d$ is referred to as the imbalance of a single ball hereafter. The rotor consists of an unbalanced rotating disk and the suspension system. The disk of mass $m_{d}$ rotates with a constant angular velocity $\omega$. The mass center $G$ of the disk is located at a distance $e$ from the disk's geometric center $C$. Therefore, the amount of imbalance of the disk is $m_{d} e$. The flexibility of the suspension system is characterized by equivalent linear springs and viscous dampers, denoted by $\left(k_{x}, k_{y}\right)$ and $\left(c_{x}, c_{y}\right)$, respectively. For simplicity, we assume that $k_{x}=k_{y}=K$ and $c_{x}=c_{y}=C$.

The governing equations of an ABS with an arbitrary number of balls have been derived in Refs. [2,5,17,19] and expressed in different dimensionless forms. For the sake of completeness, the derivation of the equations of motion is described briefly below.

The $x y$-reference frame rotates about a fixed point $O$ with the same speed as the disk. When the disk is at rest, the geometric center $C$ of the disk coincides with $O$ at which the supporting springs are undeformed. The position of $C$ is defined by the coordinates $(x, y)$ with respect to the corotating frame. The position of the $i$ th ball is given by the angle $\beta_{i}$ relative to the mass center $G$. The reason for the choice of this corotating frame is that the equations of motion expressed in this frame are autonomous.

The equations of motion are derived from Lagrange's equations given by

$$
\frac{d}{d t}\left(\frac{\partial T}{\partial \dot{q}_{k}}\right)-\frac{\partial T}{\partial q_{k}}+\frac{\partial V}{\partial q_{k}}+\frac{\partial R}{\partial \dot{q}_{k}}=0
$$

where $T$ is the kinetic energy, $V$ the potential energy, $R$ Rayleigh's dissipation function, and $q_{k}$ the generalized coordinates. The kinetic energy can be expressed as

$$
\begin{aligned}
T= & \frac{1}{2} J \omega^{2}+\frac{1}{2} M\left(\dot{x}^{2}+\dot{y}^{2}+2 \omega x \dot{y}-2 \omega \dot{x} y+\omega^{2} x^{2}+\omega^{2} y^{2}\right) \\
& +\frac{1}{2} m_{d}\left(2 \omega e \dot{y}+2 \omega^{2} e x+\omega^{2} e^{2}\right)+\frac{1}{2} m_{b} \sum_{i=1}^{3}\left[d^{2}\left(\dot{\beta}_{i}+\omega\right)^{2}\right. \\
& \left.-2 d\left(\dot{\beta}_{i}+\omega\right)(\dot{x}-\omega y) \sin \beta_{i}+2 d\left(\dot{\beta}_{i}+\omega\right)(\dot{y}+\omega x) \cos \beta_{i}\right]
\end{aligned}
$$

where $J$ is the moment of inertia of the equivalent rotor and $M$ indicates the total mass of the system. The potential energy is given by

$$
V=\frac{1}{2} K\left(x^{2}+y^{2}\right)
$$

Rayleigh's dissipation function can be represented by

$$
R=\frac{1}{2} C\left(x^{2}+y^{2}-2 \omega \dot{x} y+2 \omega x \dot{y}+\omega^{2} x^{2}+\omega^{2} y^{2}\right)+\frac{1}{2} C_{B} d^{2} \sum_{i=1}^{3} \dot{\beta}_{i}^{2}
$$

The substitution of Eqs. (2)-(4) into Eq. (1) yields the nonlinear equations of motion as follows:

$$
\begin{gathered}
M \ddot{x}-2 M \omega \dot{y}+C \dot{x}+\left(K-M \omega^{2}\right) x-C \omega y \\
-m_{b} d \sum_{i=1}^{3}\left[\ddot{\beta}_{i} \sin \beta_{i}+\left(\dot{\beta}_{i}+\omega\right)^{2} \cos \beta_{i}\right]=m_{d} e \omega^{2} \\
M \ddot{y}+2 M \omega \dot{x}+C \dot{y}+\left(K-M \omega^{2}\right) y+C \omega x \\
+m_{b} d \sum_{i=1}^{3}\left[\ddot{\beta}_{i} \cos \beta_{i}-\left(\dot{\beta}_{i}+\omega\right)^{2} \sin \beta_{i}\right]=0 \\
m_{b} d^{2} \ddot{\beta}_{i}+C_{B} d^{2} \dot{\beta}_{i}+m_{b} d\left(\ddot{y} \cos \beta_{i}+2 \omega \dot{y} \sin \beta_{i}-\omega^{2} y \cos \beta_{i}\right) \\
-m_{b} d\left(\ddot{x} \sin \beta_{i}-2 \omega \dot{x} \cos \beta_{i}-\omega^{2} x \sin \beta_{i}\right)=0, \quad i=1,2,3
\end{gathered}
$$

In order to simplify the subsequent analysis, we introduce the following dimensionless variables:

$$
x^{*}=x / e, \quad y^{*}=y / e, \quad \delta=d / e, \quad t^{*}=t \omega_{n}
$$

where $\omega_{n}=\sqrt{K / M}$ indicates the undamped natural frequency of the system when the disk is not rotating. The equations of motion in terms of the dimensionless variables defined above can be written in matrix form as

$$
\mathbf{M q} \mathbf{q}^{\prime \prime}+(\mathbf{C}+\mathbf{G}) \mathbf{q}^{\prime}+\mathbf{g}+\mathbf{f}=0
$$

where $\mathbf{q}=\left[x^{*}, y^{*}, \beta_{1}, \beta_{2}, \beta_{3}\right]^{T}$, ( )' indicates differentiation with respect to $t^{*}$, and

$\mathbf{M}=\left[\begin{array}{ccccc}1 & 0 & -\mu \eta \sin \beta_{1} & -\mu \eta \sin \beta_{2} & -\mu \eta \sin \beta_{3} \\ 0 & 1 & \mu \eta \cos \beta_{1} & \mu \eta \cos \beta_{2} & \mu \eta \cos \beta_{3} \\ -\mu \eta \sin \beta_{1} & \mu \eta \cos \beta_{1} & \mu \eta \delta & 0 & 0 \\ -\mu \eta \sin \beta_{2} & \mu \eta \cos \beta_{2} & 0 & \mu \eta \delta & 0 \\ -\mu \eta \sin \beta_{3} & \mu \eta \cos \beta_{3} & 0 & 0 & \mu \eta \delta\end{array}\right]$

$$
\mathbf{C}=\left[\begin{array}{ccccc}
2 \varsigma & 0 & 0 & 0 & 0 \\
0 & 2 \varsigma & 0 & 0 & 0 \\
0 & 0 & 2 \varsigma_{B} \mu \eta \delta & 0 & 0 \\
0 & 0 & 0 & 2 \varsigma_{B} \mu \eta \delta & 0 \\
0 & 0 & 0 & 0 & 2 \varsigma_{B} \mu \eta \delta
\end{array}\right]
$$

$\mathbf{G}=2 \Omega\left[\begin{array}{ccccc}0 & -1 & -\mu \eta \cos \beta_{1} & -\mu \eta \cos \beta_{2} & -\mu \eta \cos \beta_{3} \\ 1 & 0 & -\mu \eta \sin \beta_{1} & -\mu \eta \sin \beta_{2} & -\mu \eta \sin \beta_{3} \\ \mu \eta \cos \beta_{1} & \mu \eta \sin \beta_{1} & 0 & 0 & 0 \\ \mu \eta \cos \beta_{2} & \mu \eta \sin \beta_{2} & 0 & 0 & 0 \\ \mu \eta \cos \beta_{3} & \mu \eta \sin \beta_{3} & 0 & 0 & 0\end{array}\right]$




$$
\begin{aligned}
& \mathbf{f}=\left[\begin{array}{c}
\left(1-\Omega^{2}\right) x^{*}-2 \varsigma \Omega y^{*}-\mu \eta \Omega^{2} \sum_{i=1}^{3} \cos \beta_{i}-\mu \Omega^{2} \\
2 \varsigma \Omega x^{*}+\left(1-\Omega^{2}\right) y^{*}-\mu \eta \Omega^{2} \sum_{i=1}^{3} \sin \beta_{i} \\
\mu \eta \Omega^{2}\left(x^{*} \sin \beta_{1}-y^{*} \cos \beta_{1}\right) \\
\mu \eta \Omega^{2}\left(x^{*} \sin \beta_{2}-y^{*} \cos \beta_{2}\right) \\
\mu \eta \Omega^{2}\left(x^{*} \sin \beta_{3}-y^{*} \cos \beta_{3}\right)
\end{array}\right] \\
& \mathbf{g}=\left[\begin{array}{c}
-\mu \eta \sum_{i=1}^{3}\left(\beta_{i}^{\prime}\right)^{2} \cos \beta_{i} \\
-\mu \eta \sum_{i=1}^{3}\left(\beta_{i}^{\prime}\right)^{2} \sin \beta_{i} \\
0 \\
0 \\
0
\end{array}\right]
\end{aligned}
$$

in which

$$
\eta=\frac{m_{b} d}{m_{d} e}, \quad \Omega=\frac{\omega}{\omega_{n}}, \quad \varsigma=\frac{C}{2 M \omega_{n}}, \quad \mu=\frac{m_{d}}{M}, \quad \varsigma_{B}=\frac{C}{2 m_{b} \omega_{n}}
$$

It is worth noting that $\eta$ is the ratio of the imbalance of a single ball to that of the disk.

\section{Equilibrium Positions}

The first step in analyzing a nonlinear system is to determine the equilibrium positions. Setting $\mathbf{q}^{\prime}, \mathbf{q}^{\prime \prime}$, and $\mathbf{g}$ in Eq. (8) to zero, the location of the equilibrium position $\widetilde{\mathbf{q}}=\left[\tilde{x}, \tilde{y}, \widetilde{\beta}_{1}, \widetilde{\beta}_{2}, \widetilde{\beta}_{3}\right]^{T}$ is given by $\mathbf{f}(\widetilde{\mathbf{q}})=0$. This set of equations can be expressed explicitly as

$$
\begin{gathered}
\left(1-\Omega^{2}\right) \tilde{x}-2 \zeta \Omega \tilde{y}-\mu \eta \Omega^{2}\left(\cos \widetilde{\beta}_{1}+\cos \widetilde{\beta}_{2}+\cos \widetilde{\beta}_{3}\right)=\mu \Omega^{2} \\
2 \zeta \Omega \tilde{x}+\left(1-\Omega^{2}\right) \tilde{y}-\mu \eta \Omega^{2}\left(\sin \widetilde{\beta}_{1}+\sin \widetilde{\beta}_{2}+\sin \widetilde{\beta}_{3}\right)=0 \\
\tilde{x} \sin \widetilde{\beta}_{i}-\tilde{y} \cos \widetilde{\beta}_{i}=0, \quad i=1,2,3
\end{gathered}
$$

To solve Eqs. (10)-(12), we introduce the polar coordinates $\tilde{x}$ $=\tilde{r} \cos \tilde{\theta}$ and $\tilde{y}=\tilde{r} \sin \tilde{\theta}$ and rewrite Eq. (12) as

$$
\widetilde{r} \sin \left(\widetilde{\beta}_{i}-\tilde{\theta}\right)=0, \quad i=1,2,3
$$

The above equations require that $\sin \left(\tilde{\beta}_{i}-\tilde{\theta}\right)=0$ or $\tilde{r}=0$. The equilibrium positions associated with these two conditions are referred to as the unbalanced equilibrium positions $\left(\sin \left(\widetilde{\beta}_{i}-\widetilde{\theta}\right)=0\right)$ and perfect balancing positions $(\widetilde{r}=0)$. If the balls are indistinguishable, the unbalanced equilibrium positions can be further divided into four cases: (i) $\widetilde{\beta}_{1}=\widetilde{\beta}_{2}=\widetilde{\beta}_{3}=\tilde{\theta}$, (ii) $\widetilde{\beta}_{1}=\widetilde{\beta}_{2}=\widetilde{\beta}_{3}=\tilde{\theta}+\pi$, (iii) $\widetilde{\beta}_{1}$ $=\widetilde{\theta}+\pi, \widetilde{\beta}_{2}=\widetilde{\beta}_{3}=\widetilde{\theta}$, and (iv) $\widetilde{\beta}_{1}=\widetilde{\theta}, \widetilde{\beta}_{2}=\widetilde{\beta}_{3}=\widetilde{\theta}+\pi$. Including the solution (v) $\widetilde{r}=0$, there are totally five different cases of solutions to Eq. (13). Cases (i) and (ii) are equivalent to a single ball with mass $3 m_{b}$ located at $\widetilde{\beta}=\widetilde{\theta}$ and $\widetilde{\beta}=\tilde{\theta}+\pi$, respectively. Similarly, cases (iii) and (iv) are equivalent to a single ball with mass $m_{b}$ located at $\widetilde{\beta}=\tilde{\theta}$ and $\widetilde{\beta}=\tilde{\theta}+\pi$, respectively. Consequently, the equilibrium positions of cases (i)-(iv) can be determined from the formulas for the equilibrium positions of a one-ball balancer by substituting appropriate values to the imbalance. Since the equilibrium positions for a one-ball balancer have been analyzed comprehensively, the detailed algebraic process for determining the equilibrium positions for cases (i)-(iv) is omitted. Only the results are listed below.

(i) $\widetilde{\beta}_{1}=\widetilde{\beta}_{2}=\widetilde{\beta}_{3}=\tilde{\theta}$. The two solutions for $\widetilde{r}$ are referred to as $\widetilde{r}_{11}$ and $\tilde{r}_{12}$ and expressed as

$$
\left.\begin{array}{l}
\tilde{r}_{11} \\
\tilde{r}_{12}
\end{array}\right\}=\mathcal{K}\left[3 \eta\left(1-\Omega^{2}\right) \pm \sqrt{\mathcal{D}}\right]
$$

where

$$
\mathcal{K}=\frac{\mu \Omega^{2}}{\left(1-\Omega^{2}\right)^{2}+4 \zeta^{2} \Omega^{2}}
$$

and

$$
\mathcal{D}=\left(1-\Omega^{2}\right)^{2}+4\left(1-9 \eta^{2}\right) \zeta^{2} \Omega^{2}
$$

The corresponding $\tilde{\theta}$ is determined by

$$
\tilde{\theta}=\tan ^{-1} \frac{-2 \zeta \Omega \widetilde{r}}{\left(1-\Omega^{2}\right) \tilde{r}-3 \mu \eta \Omega^{2}}
$$

(ii) $\widetilde{\beta}_{1}=\widetilde{\beta}_{2}=\widetilde{\beta}_{3}=\widetilde{\theta}+\pi$. The two solutions for $\widetilde{r}$ are denoted by $\widetilde{r}_{21}$ and $\widetilde{r}_{22}$ and expressed as

$$
\left.\begin{array}{l}
\tilde{r}_{21} \\
\tilde{r}_{22}
\end{array}\right\}=\mathcal{K}\left[-3 \eta\left(1-\Omega^{2}\right) \pm \sqrt{\mathcal{D}}\right]
$$

where $\mathcal{K}$ and $\mathcal{D}$ are defined by Eqs. (15) and (16), respectively. The corresponding $\tilde{\theta}$ is determined by

$$
\tilde{\theta}=\tan ^{-1} \frac{-2 \zeta \Omega \widetilde{r}}{\left(1-\Omega^{2}\right) \widetilde{r}+3 \mu \eta \Omega^{2}}
$$

(iii) $\widetilde{\beta}_{1}=\tilde{\theta}+\pi, \widetilde{\beta}_{2}=\widetilde{\beta}_{3}=\tilde{\theta}$. The two solutions for $\tilde{r}$ are

$$
\left.\begin{array}{l}
\tilde{r}_{31} \\
\tilde{r}_{32}
\end{array}\right\}=\mathcal{K}\left[\eta\left(1-\Omega^{2}\right) \pm \sqrt{\mathcal{D}^{\prime}}\right]
$$

where $\mathcal{K}$ is defined by Eq. (15) and

$$
\mathcal{D}^{\prime}=\left(1-\Omega^{2}\right)^{2}+4\left(1-\eta^{2}\right) \zeta^{2} \Omega^{2}
$$

The corresponding $\tilde{\theta}$ is determined by

$$
\tilde{\theta}=\tan ^{-1} \frac{-2 \zeta \Omega \widetilde{r}}{\left(1-\Omega^{2}\right) \tilde{r}-\mu \eta \Omega^{2}}
$$

(iv) $\widetilde{\beta}_{1}=\widetilde{\theta}, \widetilde{\beta}_{2}=\widetilde{\beta}_{3}=\tilde{\theta}+\pi$. The two solutions for $\widetilde{r}$ are

$$
\left.\begin{array}{l}
\tilde{r}_{41} \\
\tilde{r}_{42}
\end{array}\right\}=\mathcal{K}\left[-\eta\left(1-\Omega^{2}\right) \pm \sqrt{\mathcal{D}^{\prime}}\right]
$$

where $\mathcal{K}$ and $\mathcal{D}^{\prime}$ are defined by Eqs. (15) and (21), respectively. The corresponding $\tilde{\theta}$ is determined by

$$
\tilde{\theta}=\tan ^{-1} \frac{-2 \zeta \Omega \widetilde{r}}{\left(1-\Omega^{2}\right) \widetilde{r}+\mu \eta \Omega^{2}}
$$

In the above four cases, the effects of the three balls are equivalent to a single ball with proper mass located at $\widetilde{\beta}_{\text {eq }}$ Hereafter, the value of $\widetilde{\beta}_{\text {eq }}$ associated with $\widetilde{r}_{i j}$ is denoted by $\tilde{\beta}_{i j}$ and is used to identify the equilibrium positions of the three balls. For example, when $\tilde{r}=\widetilde{r}_{3 j}$, the system is equivalent to a single ball with mass $m_{b}$ located at $\widetilde{\beta}_{3 j}=\tilde{\theta}$, where $\tilde{\theta}$ is determined by substituting $\widetilde{r}_{3 j}$ into Eq. (22). In this case, more specifically, two balls stick together at $\widetilde{\beta}=\widetilde{\beta}_{3 j}$ while the other ball is located at $\widetilde{\beta}=\widetilde{\beta}_{3 j}+\pi$. Similarly, when $\widetilde{r}=\widetilde{r}_{4 j}$, one ball is located at $\widetilde{\beta}=\tilde{\theta}$ while the other two balls stick together at $\widetilde{\beta}=\widetilde{\beta}_{4 j}=\tilde{\theta}+\pi$, where $\tilde{\theta}$ is determined 
by substituting $\tilde{r}_{4 j}$ into Eq. (24). Then we study the equilibrium positions associated with case (v), $\tilde{r}=0$, in detail.

(v) $\tilde{r}=0$. In this case the system is perfectly balanced and has no residual vibration. Substituting $\tilde{x}=\tilde{y}=0$ into Eqs. (10) and (11) yields

$$
\begin{gathered}
\cos \widetilde{\beta}_{1}+\cos \widetilde{\beta}_{2}+\cos \widetilde{\beta}_{3}=-1 / \eta \\
\sin \widetilde{\beta}_{1}+\sin \widetilde{\beta}_{2}+\sin \widetilde{\beta}_{3}=0
\end{gathered}
$$

The positions of the three balls cannot be determined uniquely from these two equations. However, if the position of one of the balls is given, say, $\widetilde{\beta}_{3}$, the positions of the other two balls, $\widetilde{\beta}_{1}$ and $\widetilde{\beta}_{2}$, can be expressed in terms of $\widetilde{\beta}_{3}$. When the position of the third ball is specified, the system can be treated as a "new disk" packed with a two-ball balancer, where the new disk is composed of the original disk and the third ball. Since the perfect balancing positions of a disk packed with a two-ball balancer have been studied thoroughly, all we need to do is to determine the imbalance of the new disk and then substitute appropriate values of the imbalance ratio into the formulas for the equilibrium positions of a two-ball balancer. Let $\mathbf{r}_{G}$ and $\mathbf{r}_{B 3}$ indicate the position vectors of the mass center of the original disk and the third ball, respectively. With respect to the corotating reference frame, $\mathbf{r}_{G}=e \hat{\mathbf{i}}$ and $\mathbf{r}_{B 3}$ $=d\left(\cos \widetilde{\beta}_{3} \hat{\mathbf{i}}+\sin \widetilde{\beta}_{3} \hat{\mathbf{j}}\right)$. The position vector $\mathbf{r}_{G^{\prime}}$ of the center of mass of the new disk can be expressed as

$$
\mathbf{r}_{G^{\prime}}=\frac{1}{m_{d}+m_{b}}\left(m_{b} \mathbf{r}_{B 3}+m_{d} \mathbf{r}_{G}\right)=e^{\prime}(\cos \phi \hat{\mathbf{i}}+\sin \phi \hat{\mathbf{j}})
$$

where

$$
e^{\prime}=\frac{m_{d} e}{m_{b}+m_{d}} \sqrt{\eta^{2}+2 \eta \cos \widetilde{\beta}_{3}+1}
$$

and

$$
\phi=\tan ^{-1}\left(\frac{\eta \sin \widetilde{\beta}_{3}}{\eta \cos \widetilde{\beta}_{3}+1}\right)
$$

Thus the imbalance ratio of the new disk is given by

$$
\eta^{\prime}=\frac{m_{b} d}{\left(m_{b}+m_{d}\right) e^{\prime}}
$$

It is well known that for a two-ball balancer, perfect balancing is possible only if the imbalance ratio is no less than 0.5. Consequently, for the new disk to be perfectly balanced,

$$
\eta^{\prime}=\frac{m_{b} d}{\left(m_{b}+m_{d}\right) e^{\prime}} \geqslant \frac{1}{2}
$$

By substituting Eq. (28) into Eq. (31) and rearranging terms, we obtain the condition for perfect balancing for the three-ball balancer as

$$
\cos \widetilde{\beta}_{3} \leqslant A(\eta)=\frac{3 \eta^{2}-1}{2 \eta}
$$

Based on this result, we can determine the restriction on $\eta$ for perfect balancing and the corresponding range of $\widetilde{\beta}_{3}$. By noticing that $A(\eta)$ is strictly increasing for $\eta>0$ and using the facts $A(1 / 3)=-1, A(1 / \sqrt{3})=0$, and $A(1)=1$, we have the following results.

(1) For $\eta<1 / 3, A(\eta)<-1$. In this case, the total imbalance of the three balls is less than that of the disk. Hence perfect balancing is impossible.

(2) For $1 / 3 \leqslant \eta \leqslant 1,-1 \leqslant A(\eta) \leqslant 1$. From Eq. (32), -1 $\leqslant \cos \widetilde{\beta}_{3} \leqslant A(\eta)$. Hence, $\widetilde{\beta}_{3}$ must lie in the range

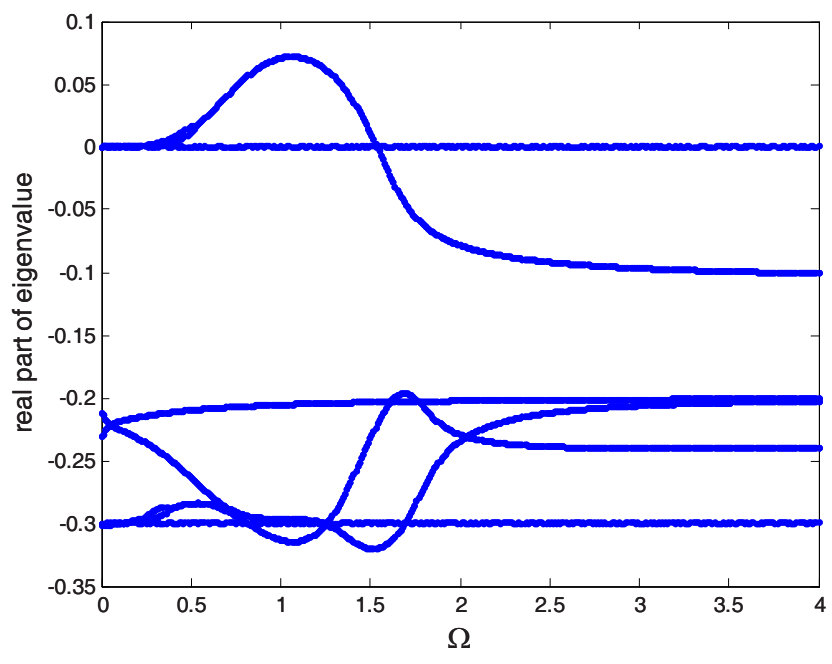

Fig. 2 Variation of the real parts of eigenvalues with the rotational speed for a perfect balancing position of a three-ball balancer with $\eta=0.4$

$$
\left[\beta^{*}, 2 \pi-\beta^{*}\right], \quad \text { where } \beta^{*}=\cos ^{-1} A(\eta)
$$

Since the balls are indistinguishable, all three balls must lie in this range.

(3) For $\eta>1, A(\eta)>1$. As a result, there is no restriction on the position of the balls.

The above discussion indicates that for $\eta \geqslant 1 / 3$, perfect balancing is possible. In this case, it is not difficult to show that the positions of the other two balls can be expressed in terms of $\widetilde{\beta}_{3}$ as

$$
\begin{aligned}
& \tilde{\beta}_{1}=\pi-\cos ^{-1}\left(\frac{\sqrt{\eta^{2}+2 \eta \cos \widetilde{\beta}_{3}+1}}{2 \eta}\right)+\tan ^{-1}\left(\frac{\eta \sin \widetilde{\beta}_{3}}{\eta \cos \widetilde{\beta}_{3}+1}\right) \\
& \tilde{\beta}_{2}=\pi+\cos ^{-1}\left(\frac{\sqrt{\eta^{2}+2 \eta \cos \widetilde{\beta}_{3}+1}}{2 \eta}\right)+\tan ^{-1}\left(\frac{\eta \sin \widetilde{\beta}_{3}}{\eta \cos \widetilde{\beta}_{3}+1}\right)
\end{aligned}
$$

In summary, the system is perfectly balanced if $\widetilde{\beta}_{1}$ and $\widetilde{\beta}_{2}$ are given by Eq. (34) and $\widetilde{\beta}_{3}$ lies in the range

$$
\left[\beta^{*}, 2 \pi-\beta^{*}\right]
$$

where

$$
\beta^{*}=\left\{\begin{array}{cc}
\cos ^{-1}\left(\frac{3 \eta^{2}-1}{2 \eta}\right), & \frac{1}{3} \leqslant \eta \leqslant 1 \\
0, & 1<\eta
\end{array}\right.
$$

\section{Stability Analysis}

The stability of an equilibrium position can be determined by the eigenvalues of the associated linearized system if all the eigenvalues have negative real parts or if at least one of the eigenvalues has a positive real part. The linearization fails when the linearized system has some eigenvalues with zero real parts and no eigenvalues with positive real parts. For unbalanced equilibrium positions, i.e., $\widetilde{r} \neq 0$, none of the eigenvalues have zero real part. In this case, the stability of the equilibrium position can be determined using the Routh criterion. For perfect balancing positions, by contrast, there may exist eigenvalues with zero real parts. Figure 2 is a typical result of a three-ball balancer showing the variation of the real parts of the eigenvalues of a perfect balancing position with the rotational speed. As can be seen from the figure, when the rotational speed is lower than about 1.51 , one eigenvalue 
has a positive real part. Hence, the perfect balancing position is unstable for $\Omega<1.51$. On the other hand, when the rotational speed is higher than 1.51, one eigenvalue is zero while the other eigenvalues have negative real parts. Since none of the eigenvalues in this speed range has a positive real part, the stability of the perfect balancing position cannot be determined from the linearized system. Therefore, in the present study, the stability of a perfect balancing position is determined from its steady state behaviors subjected to disturbances. We consider disturbances in each coordinate direction of the state space in sequence, one direction at a time. The steady state behavior is obtained by integrating numerically the nonlinear governing equation (Eq. (8)) for a long time until the transient vibration dies away. A perfect balancing position is stable if the steady state solutions under all disturbances approach the initial position; otherwise, it is unstable.

There is another issue regarding the stability of the perfect balancing positions needed to be addressed. For a two-ball balancer, each ball should stay at a specific position when the system is perfectly balanced. In other words, there is a unique perfect balancing position. By contrast, for a three-ball balancer, there are infinitely many perfect balancing positions. The system is perfectly balanced if all the balls stay in the angular range defined by Eq. (35) and their positions satisfy Eq. (34). From the point of view of practical application, the exact locations of the balls are not important as long as the disk is perfectly balanced. Hence, when studying the stability of the perfect balancing positions, we divide evenly the possible angular range of the balls, as defined by Eq. (35), into several small intervals. By setting $\widetilde{\beta}_{3}$ to the central value of each small interval and determining $\widetilde{\beta}_{1}$ and $\widetilde{\beta}_{2}$ from Eq. (34), we get a set of equilibrium positions. The stability of each equilibrium position in the set is examined by the method discussed in the preceding paragraph. In this way, the stable region of each equilibrium position in a parameter plane can be identified. The stable region of $\widetilde{r}=0$ is taken as the union of the stable regions of the set of equilibrium positions examined.

Previous investigations on the ball-type automatic balancer indicate that in addition to the imbalance ratio $\eta$, the rotational speed $\Omega$, damping ratio $\zeta$ of the suspension, and damping ratio $\zeta_{b}$ between the balls and the orbit are important parameters influencing the performance of the automatic balancer. Therefore, we study the stability of the perfect balancing positions in the $\zeta-\Omega$ plane point by point for different values of $\eta$ and $\zeta_{b}$. For a specified value of $\eta$, stable regions for $\tilde{r}=0$ in the $\zeta-\Omega$ plane for various values of $\zeta_{b}$ are identified, and the results are compared with those of a two-ball balancer.

\section{Results and Discussion}

Figure 3 shows the variation of the equilibrium positions with the rotational speed for $\eta=0.2$. As discussed before, for $\eta<1 / 3$, perfect balancing can not be achieved. Hence, for $\eta=0.2$, all the equilibrium radii are nonzero. In addition, the equilibrium radii $\tilde{r}_{i 2}, i=1-4$, are negative. Since negative radii are unrealistic, all the negative equilibrium radii $\widetilde{r}_{i 2}$ and the associate $\widetilde{\beta}$ 's are not shown in Fig. 3. For simplicity, from now on, we use the equilibrium radius to identify the corresponding equilibrium configuration. For example, $\widetilde{r}_{11}$ indicates the equilibrium configuration in which the three balls stick together at $\widetilde{\beta}_{11} ; \widetilde{r}_{21}$ indicates the equilibrium configuration in which the three balls stick together at $\widetilde{\beta}_{21}$. On the other hand, $\widetilde{r}_{31}$ indicates the equilibrium configuration in which one ball is at $\widetilde{\beta}_{31}+\pi$ and the other two balls are at $\widetilde{\beta}_{31} ; \widetilde{r}_{41}$ indicates the equilibrium configuration in which one ball is at $\widetilde{\beta}_{41}-\pi$ and the other two balls are at $\widetilde{\beta}_{41}$. The meaning of $\widetilde{\beta}_{i j}$ is explained in the paragraph below Eq. (24). The circles in Fig. 3 denote the steady state responses obtained from numerical integration. The results imply that among all the equilibrium positions, $\widetilde{r}_{11}$ may be the only stable equilibrium position at the spe-
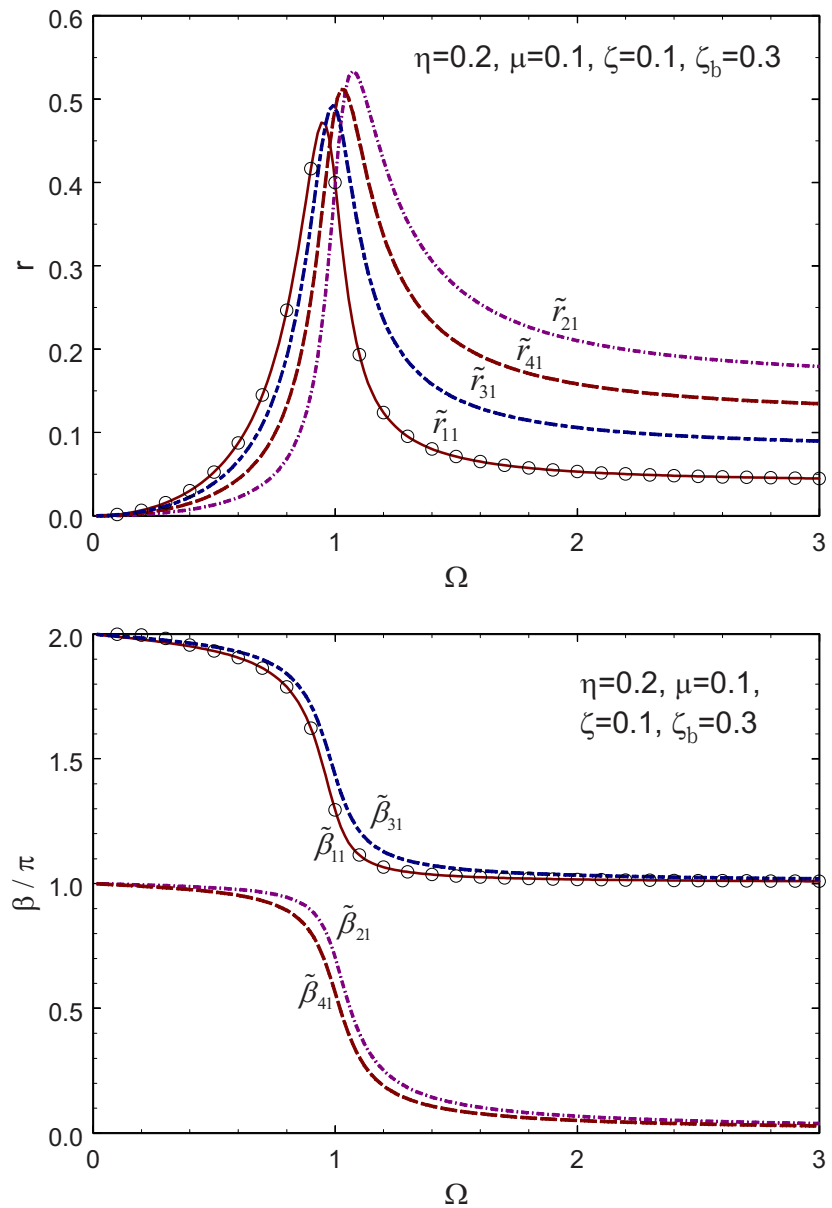

Fig. 3 Variation of the equilibrium positions with the rotational speed for $\eta=0.2$

cific values of parameters. Then we check the stability of $\tilde{r}_{11}$ with the variations of $\zeta, \zeta_{b}$, and $\Omega$. The stability of $\tilde{r}_{11}$ is determined by the eigenvalues of the corresponding linearized system.

The stable regions of $\widetilde{r}_{11}$ in the $\zeta-\Omega$ plane at $\eta=0.2$ for different values of $\zeta_{b}$ are shown in Fig. 4. Each bell-shaped curve indicates the boundary of the unstable region of $\widetilde{r}_{11}$ for the corresponding value of $\zeta_{b}$. Specifically, inside the area enclosed by the bellshaped curve and the horizontal axis, $\widetilde{r}_{11}$ is unstable. For example,

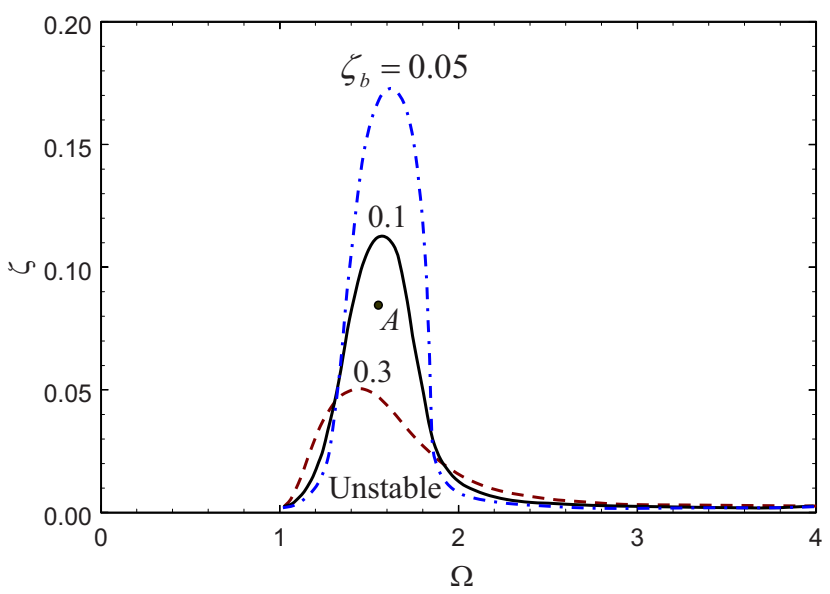

Fig. 4 Stable region of $\tilde{r}_{11}$ at $\eta=0.2$ for various values of $\zeta_{b}$ 

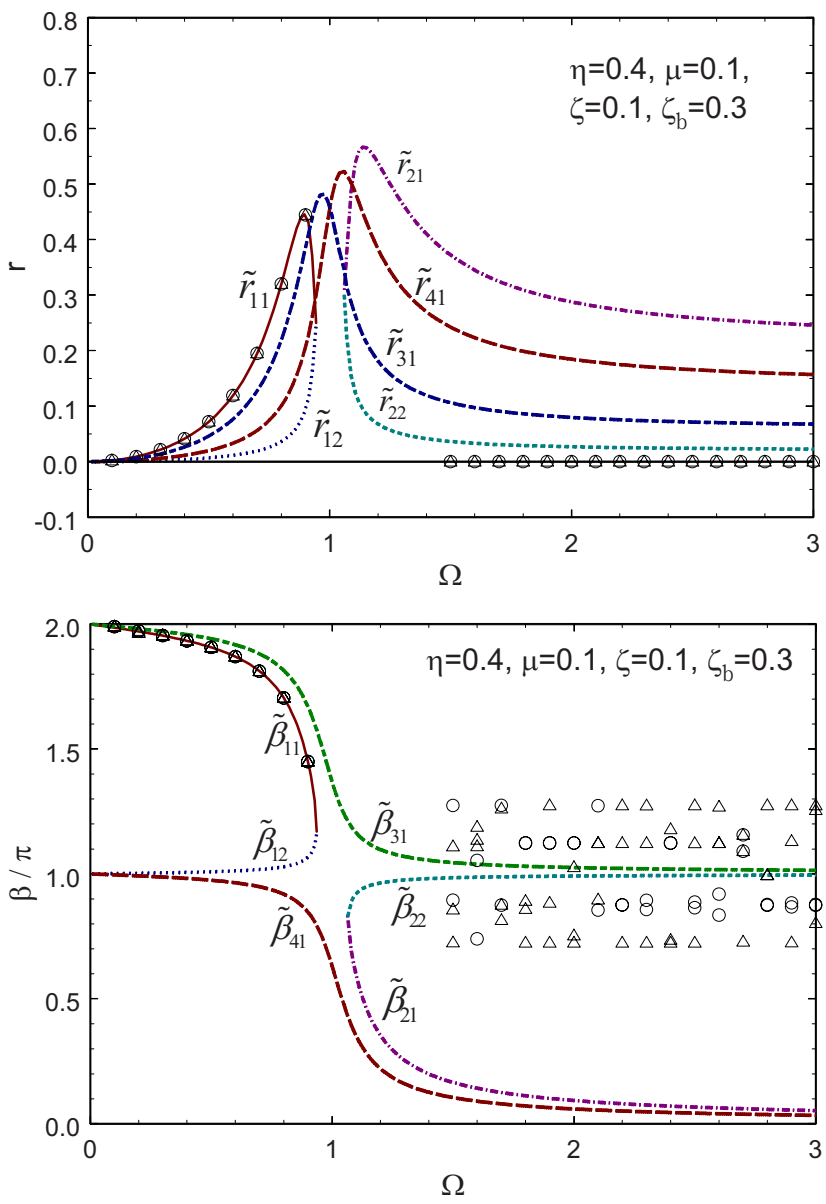

Fig. 5 Variation of the equilibrium positions with the rotational speed for $\eta=0.4$

at point $A, \widetilde{r}_{11}$ is stable for $\zeta_{b}=0.3$ but unstable for $\zeta_{b}=0.1$. The results indicate that the unstable region for $\widetilde{r}_{11}$ decreases with increasing $\zeta_{b}$.

Figure 5 shows the variation of the equilibrium positions with the rotational speed for $\eta=0.4$; different lines indicate different equilibrium configurations. For the meanings of $\widetilde{r}_{i j}$ and $\widetilde{\beta}_{i j}$, refer to the first paragraph of this section. The circles and triangles denote steady state results obtained by numerical integration from different initial conditions. The results of numerical integration indicate that all equilibrium positions, except $\widetilde{r}_{11}$ and $\widetilde{r}=0$, are unstable. Furthermore, $\widetilde{r}_{11}$ is stable when the rotational speed is less than about 0.9 , while $\tilde{r}=0$ is stable when the rotational speed is higher than about 1.42. As can be seen from the bottom of Fig. 5 , when $\widetilde{r}=0$, the steady-state positions of the balls depend on the initial conditions. To clearly illustrate the dependence of the steady-state positions on the initial conditions, the responses of the system starting from two different sets of initial conditions are plotted in Fig. 6. The two sets of initial conditions are (1) $x=y$ $=0, \beta_{1}=0.01 \pi, \beta_{2}=0.02 \pi, \beta_{3}=0.03 \pi$, and $\dot{x}=\dot{y}=\dot{\beta}_{i}=0$ and (2) $x=y=0, \beta_{1}=0.30 \pi, \beta_{2}=0.32 \pi, \beta_{3}=0.34 \pi$, and $\dot{x}=\dot{y}=\dot{\beta}_{i}=0$. The results indicate that in the steady state, the system is perfectly balanced but the balls occupy two different sets of positions. Then we proceed to compare the stable regions of $\tilde{r}=0$ for a three-ball balancer to those for a two-ball balancer. To make the comparison of stable regions meaningful, the total imbalance ratio (the ratio of the imbalance of all the balls to that of the disk) should be the same. Recall that $\eta$ indicates the ratio of the imbalance of a single ball to that of the disk. The total imbalance ratio $\eta_{\text {total }}$ can be expressed as
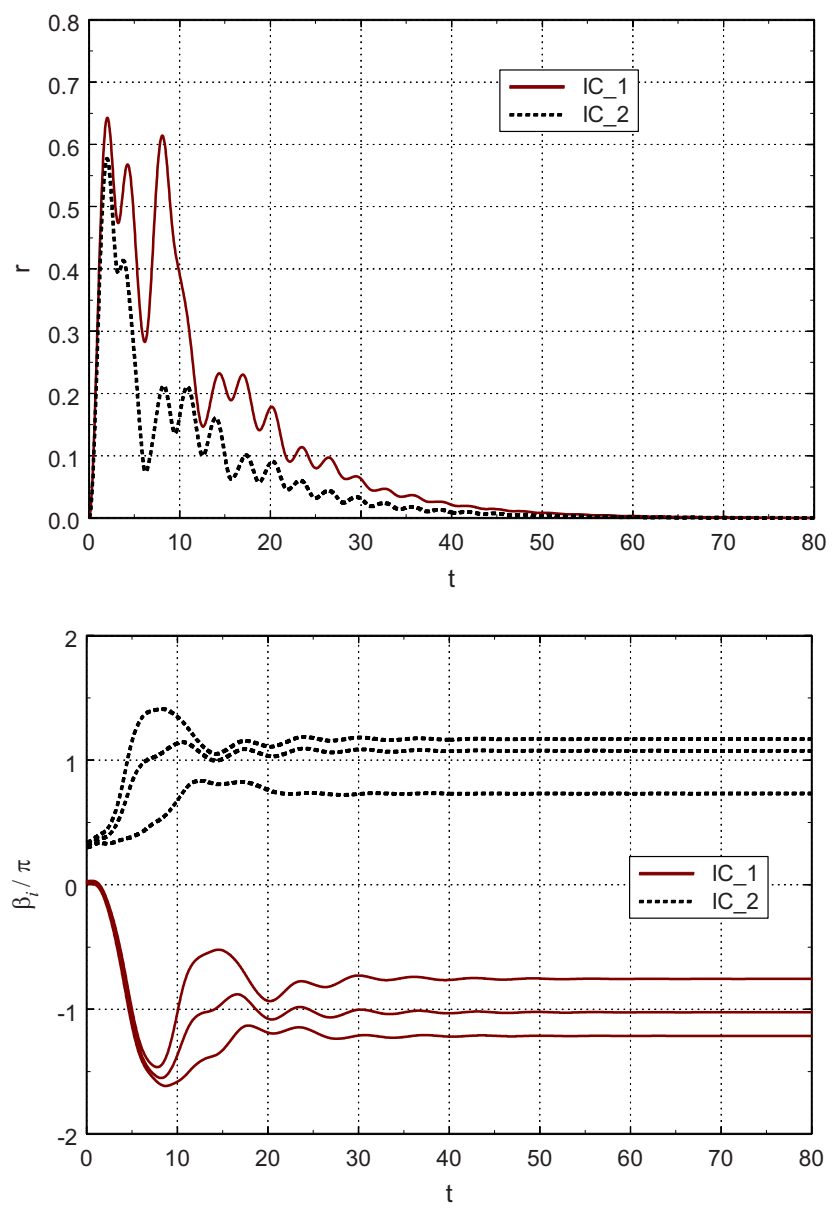

Fig. 6 Responses starting from two different sets of initial conditions for $\eta=0.4$ and $\Omega=2$

$$
\eta_{\text {total }}=2 \eta_{\text {two }}=3 \eta_{\text {three }}
$$

where $\eta_{\text {two }}$ and $\eta_{\text {three }}$ are the imbalance ratios of a single ball for the two-ball and three-ball balancers, respectively.

Figure 7 compares the stable regions of $\tilde{r}=0$ for the three-ball and two-ball balancers in the $\zeta-\Omega$ plane at $\eta_{\text {total }}=1.2$. The solid and dashed lines indicate the boundaries of the stable regions for the three-ball and two-ball balancers, respectively. Inside the area to the right and below the solid/dashed line, $\widetilde{r}=0$ is stable for the

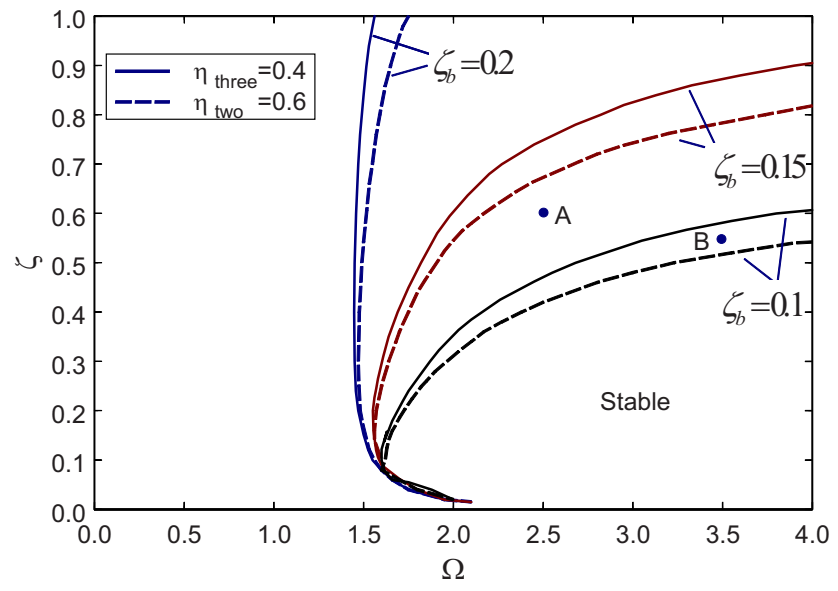

Fig. 7 Comparison of the stable regions of $\tilde{r}=0$ for the threeball (solid) and two-ball (dashed) balancers at $\eta_{\text {total }}=1.2$ 


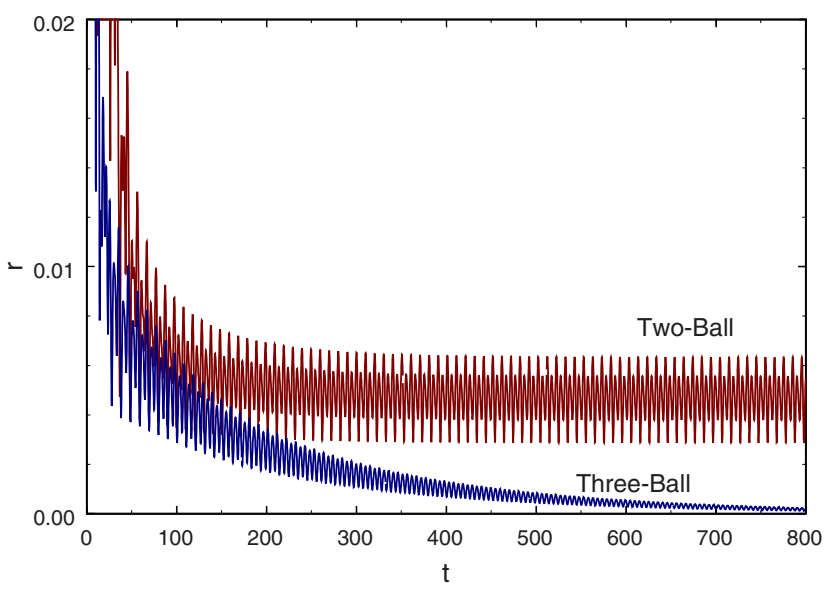

Fig. 8 Radial vibrations of the two-ball and three-ball balancers at point B in Fig. 7 for $\zeta_{b}=0.1$

associated value of $\zeta_{b}$. For example, when $\zeta_{b}=0.1, \tilde{r}=0$ is unstable at point $A$ for both the two-ball and three-ball balancers. On the other hand, at point $B, \widetilde{r}=0$ is stable for the three-ball balancer but unstable for the two-ball balancer. Time responses for the two-ball and three-ball balancers associated with point $B$ for $\zeta_{b}$ $=0.1$ are shown in Fig. 8. The results verify that $\tilde{r}=0$ is unstable for the two-ball balancer but stable for the three-ball balancer. Figure 9 compares the stable regions of the three-ball and two-ball balancers in the $\zeta-\Omega$ plane for $\eta_{\text {total }}=2.4$. Figures 7 and 9 show that the three-ball balancer has larger stable regions than the twoball balancer under the conditions examined. This, in turn, implies that the robustness of an ABS can be enhanced by employing more balls if the other parameters are fixed.

\section{Conclusion}

Ball-type automatic balancers are employed widely by the optical disk drive industry to suppress the vibrations induced by the eccentricity of the optical disk. Proper conditions under which the imbalance vibrations can be suppressed perfectly are closely related to the stability of the perfect balancing positions. Although much work has been done on the analysis of the stability of balltype automatic balancers, the effects of the number of balls on the performance of the balancer have not been clearly examined. This paper investigates the stability of the perfect balancing positions of a three-ball automatic balancer. Closed-form formulas for the

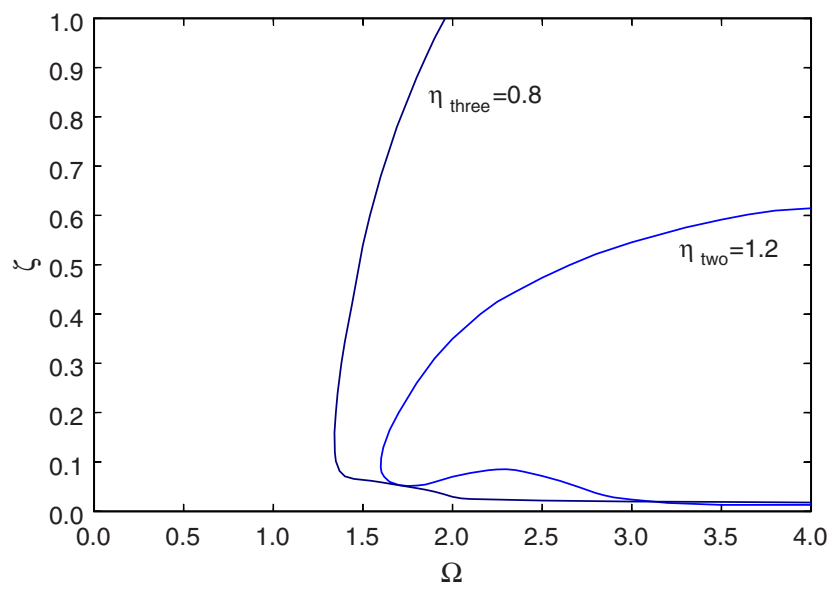

Fig. 9 Comparison of the stable regions of $\tilde{r}=0$ for the two-ball and three-ball balancers at $\eta_{\text {total }}=2.4$ equilibrium positions are derived, and the conditions for perfect balancing are examined. The results show that, to achieve perfect balancing, the imbalance ratio of a single ball should be no less than one-third. Moreover, the balls should all stay in an angular range relative to the mass center of the disk. The stability of the perfect balancing positions is studied numerically and the stable regions in a parameter plane are identified. The results indicate that the three-ball balancer has larger stable regions compared with the two-ball balancer in the parameter space examined. Therefore increasing the number of balls may enhance the robustness of the ball-type automatic balancer.

\section{Acknowledgment}

This work was supported by the National Science Council of R.O.C. under Grant No. NSC94-2212-E-002-033.

\section{References}

[1] Bövik, P., and Högfors, C., 1986, "Autobalancing of Rotors," J. Sound Vib., 111(3), pp. 429-440.

[2] Lee, J., and Van Moorhem, W. K., 1996, "Analytical and Experimental Analysis of a Self-Compensating Dynamic Balancer in a Rotating Mechanism," ASME J. Dyn. Syst., Meas., Control, 118, pp. 468-475.

[3] Rajalingham, C., Bhat, R. B., and Rakheja, S., 1998, "Automatic Balancing of Flexible Vertical Rotors Using a Guided Ball,” Int. J. Mech. Sci., 40(9), pp. 825-834.

[4] Hwang, C.-H., and Chung, J., 1999, "Dynamic Analysis of an Automatic Ball Balancer With Double Races," JSME Int. J., Ser. C, 42(2), pp. 265-272.

[5] Chung, J., and Ro, D. S., 1999, "Dynamic Analysis of an Automatic Dynamic Balancer for Rotating Mechanisms," J. Sound Vib., 228(5), pp. 1035-1056.

[6] Kang, J. R., Chao, C. P., Huang, C. L., and Sung, C. K., 2001, "The Dynamics of a Ball-Type Balancer System Equipped With a Pair of Free-Moving Balancing Masses," ASME J. Vibr. Acoust., 123(4), pp. 456-465.

[7] Kim, W., and Chung, J., 2002, "Performance of Automatic Ball Balancers on Optical Disc Drives," Proc. Inst. Mech. Eng., Part C: J. Mech. Eng. Sci., 216(11), pp. 1071-1080.

[8] Huang, W. Y., Chao, C. P., Kang, J. R., and Sung, C. K., 2002, "The Application of Ball-Type Balancers for Radial Vibration Reduction of High-Speed Optic Disk Drives,” J. Sound Vib., 250(3), pp. 415-430.

[9] Lu, C. J., 2006, "Stability Analysis of a Single-Ball Automatic Balancer," ASME J. Vibr. Acoust., 128(1), pp. 122-125.

[10] Wang, M.-C., and Lu, C.-J., 2007, "Dynamic Characteristics of a One-Unit Ball-Rod-Spring Balancer," Trans. ASME, J. Vib. Acoust., 129(4), pp. 520524.

[11] Chung, J., and Jang, I., 2003, "Dynamic Response and Stability Analysis of an Automatic Ball Balancer for a Flexible Rotor," J. Sound Vib., 259(1), pp. $31-43$

[12] Kim, W., Lee, D.-J., and Chung, J., 2005, “Three-Dimensional Modelling and Dynamic Analysis of an Automatic Ball Balancer in an Optical Disk Drive," J. Sound Vib., 285(3), pp. 547-569.

[13] Chao, P. C. P., Huang, Y.-D., and Sung, C.-K., 2003, "Non-Planar Dynamic Modeling for the Optical Disk Drive Spindles Equipped With an Automatic Balancer," Mech. Mach. Theory, 38(11), pp. 1289-1305.

[14] Chao, P. C. P., Sung, C. K., Wu, S. T., and Huang, J. S., 2007, "Nonplanar Modeling and Experimental Validation of a Spindle-Disk System Equipped With an Automatic Balancer System in Optical Disk Drives," Microsyst. Technol., 13(8-10), pp. 1227-1239.

[15] Chao, P. C. P., Sung, C.-K., and Wang, C.-C., 2005, "Dynamic Analysis of the Optical Disk Drives Equipped With an Automatic Ball Balancer With Consideration of Torsional Motions," ASME J. Appl. Mech., 72(6), pp. 826-842.

[16] Chao, P. C. P., Yo, B. C., Sung, C. K., and Chiug, C. W., 2006, "Nonlinear Dynamic Effects of Damping Washers on the Performance of Automatic Ball Balancers in Optical Disc Drives," Int. J. Nonlinear Sci. Numer. Simul., 7(3), pp. 275-278.

[17] Rajalingham, C., and Bhat, R. B., 2006, "Complete Balancing of a Disk Mounted on a Vertical Cantilever Shaft Using a Two Ball Automatic Balancer,” J. Sound Vib., 290(1-2), pp. 169-191.

[18] Green, K., Champneys, A. R., and Friswell, M. I., 2006, "Analysis of the Transient Response of an Automatic Dynamic Balancer for Eccentric Rotors," Int. J. Mech. Sci., 48(3), pp. 274-293.

[19] Green, K., Champneys, A. R., and Lieven, N. J., 2006, "Bifurcation Analysis of an Automatic Dynamic Balancing Mechanism for Eccentric Rotors," J. Sound Vib., 291(3-5), pp. 861-881.

[20] Van de Wouw, N., Van den Heuvel, M. N., Nijmeijer, H., and Van Rooij, J. A., 2005, "Performance of an Automatic Ball Balancer With Dry Friction," Int. J. Bifurcation Chaos Appl. Sci. Eng., 15(1), pp. 65-82.

[21] Yang, Q., Ong, E.-H., Sun, J., Guo, G., and Lim, S.-P., 2005, "Study on the Influence of Friction in an Automatic Ball Balancing System," J. Sound Vib., 285(1-2), pp. 73-99.

[22] Chao, P. C. P., Sung, C.-K., and Leu, H.-C., 2005, "Effects of Rolling Friction of the Balancing Balls on the Automatic Ball Balancer for Optical Disk Drives,” ASME J. Tribol., 127(4), pp. 845-856. 